\title{
Construção de diagnósticos de enfermagem para pessoas com lesão medular em reabilitação
}

\author{
Construction of nursing diagnoses for people with spinal cord injury in rehabilitation \\ Construcción de diagnósticos de enfermería para personas con lesión medular \\ en rehabilitación
}

Como citar este artigo:

Clares JWB, Guedes MVC, Freitas MC. Construction of nursing diagnoses for people with spinal cord injury in rehabilitation. Rev Esc Enferm USP 2021;55:e03750. https://doi.org/10.1590/S1980-220X2020038403750

\section{Jorge Wilker Bezerra Clares ${ }^{1}$}

Maria Vilaní Cavalcante Guedes ${ }^{2}$

Maria Célia de Freitas ${ }^{2}$

${ }^{1}$ Rede SARAH de Hospitais de Reabilitação, São Luís, MA, Brasil.

${ }^{2}$ Universidade Estadual do Ceará, Centro de Ciências da Saúde, Programa de

Pós-Graduação Cuidados Clínicos em

Enfermagem e Saúde, Fortaleza, CE, Brasil.

\begin{abstract}
Objective: To construct nursing diagnosis statements of the International Classification for Nursing Practice $\left(\mathrm{ICNP}^{\oplus}\right)$ for the spinal cord rehabilitation specialty based on the Callista Roy Adaptation Model. Method: Descriptive study developed in sequential steps of identification and cross-mapping of terms relevant to care with the health priority, construction and cross-mapping of nursing diagnosis statements based on the ICNP ${ }^{\oplus}$, and categorization of diagnoses according to adaptive modes. Results: Ninety-two statements of nursing diagnoses were developed, of which 66 were classified in the Physiological Mode, 12 in Functional Role Mode, 10 in Self-Concept Mode and four in Interdependence Mode. Conclusion: The nursing diagnoses developed portray focuses of attention for nursing care to people with spinal cord injury in rehabilitation and contribute to the direction of nursing care actions in a systematic, individualized and resolutive way.
\end{abstract}

\section{DESCRIPTORS}

Nursing; Nursing Diagnosis; Terminology; Classification; Rehabilitation; Spinal Cord Injuries. 


\section{INTRODUÇÃO}

A lesão medular (LM) é uma síndrome neurológica incapacitante com importantes repercussões fisiológicas, emocionais, sociais e econômicas, que requer um programa de reabilitação complexo e diversificado, visando a adaptação às mudanças pessoais e ambientais, de acordo com o potencial de recuperação funcional do indivíduo, de forma a possibilitar sua reinserção social e qualidade de vida ${ }^{(1)}$.

$\mathrm{O}$ enfermeiro tem papel de destaque no processo de reabilitação das pessoas com LM, como membro da equipe interdisciplinar. Esse profissional é responsável por identificar as necessidades de cuidado e elaborar diagnósticos de enfermagem, mediante adoção de um método de raciocínio crítico e julgamento clínico que servirá de base para selecionar intervenções que resultem na promoção da saúde desses sujeitos ${ }^{(2)}$.

Para direcionar o cuidado de enfermagem, esses profissionais devem lançar mão de modelos conceituais e de linguagem padronizada para guiar a implementação do processo de enfermagem. Dentre os sistemas de classificação de enfermagem existentes, destaca-se a Classificação Internacional para a Prática de Enfermagem $\left(\mathrm{CIPE}^{\varpi}\right)$, por representar os conceitos de enfermagem (diagnósticos, resultados e intervenções) em padrão internacional, e possibilitar a estruturação de subconjuntos terminológicos direcionados a áreas específicas da prática clínica ${ }^{(3)}$.

Em apoio aos sistemas de classificação, os enfermeiros dispõem de diversos modelos conceituais para o desenvolvimento de ações fundamentadas em conhecimento científico. Dentre eles, o Modelo de Adaptação de Callista Roy tem sido amplamente utilizado na prática clínica de enfermagem. A teórica define a pessoa como um sistema holístico e adaptável, que emite respostas adaptativas ou ineficientes frente aos estímulos recebidos; saúde é um estado e um processo de tornar-se uma pessoa total e integrada; o ambiente é composto por todas as condições, circunstâncias e influências que afetam o desenvolvimento e o comportamento da pessoa; e a meta da enfermagem é promover adaptação nos modos adaptativos fisiológico, autoconceito, papel funcional e interdependência ${ }^{(4-5)}$. Esse modelo teórico possibilita a operacionalização do processo de enfermagem em todas as suas etapas, visando a promoção da adaptação do indivíduo nos quatro modos adaptativos e pode contribuir de forma eficaz para a reabilitação e qualidade de vida das pessoas com LM.

Estudo aponta a necessidade de elucidar os focos da prática da Enfermagem para desenvolver estratégias de cuidado que visem a melhor adaptação da pessoa com LM ao tratamento reabilitador ${ }^{(6)}$. Portanto, é relevante ter clareza dos diagnósticos de enfermagem que podem ocorrer nesse grupo, a fim de subsidiar o planejamento da assistência de enfermagem.

A construção de diagnósticos de enfermagem da $\mathrm{CIPE}^{\circledast}$ para pessoas com LM em reabilitação atende às recomendações internacionais ${ }^{(3,7-8)}$ e resultará em um instrumento tecnológico facilitador para a prática sistematizada de enfermagem, apoiada em referencial teórico apropriado ao contexto do cuidado, raciocínio clínico e em um vocabulário padronizado de enfermagem, além de contribuir para aprimorar a prática clínica e oferecer maior visibilidade à profissão. Em face do exposto, o presente estudo objetivou construir enunciados de diagnósticos de enfermagem da $\mathrm{CIPE}^{\oplus}$ para a especialidade de reabilitação em LM, com base no Modelo de Adaptação de Roy.

\section{MÉTODO}

\section{TIPO DE ESTUdo}

Pesquisa descritiva, que seguiu os procedimentos metodológicos adaptados de estudos terminológicos ${ }^{(3,7-8)}$.

\section{Coleta, AnÁlise e tratamento dos Dados}

A pesquisa foi realizada no período de maio de 2016 a janeiro de 2017, estruturada em cinco etapas: 1) coleta de termos relevantes para a prática clínica de enfermagem na reabilitação de pessoas com LM; 2) mapeamento dos termos identificados com os termos da CIPE ; 3) construção dos enunciados de diagnósticos de enfermagem a partir dos termos identificados; 4) mapeamento dos enunciados de diagnósticos construídos com os constantes da CIPE; ; 5) categorização dos diagnósticos de enfermagem segundo o Modelo de Adaptação de Roy.

$\mathrm{Na}$ primeira etapa, foram realizadas a análise do documento Diretrizes de Atenção à Pessoa com Lesão Medular, do Ministério da Saúde ${ }^{(9)}$ e a extração dos termos considerados clínica e culturalmente relevantes para a prática de enfermagem em reabilitação. Esse documento foi escolhido por ser um guia de referência às equipes multiprofissionais de saúde para o cuidado à saúde da pessoa com LM nos diferentes cenários de atenção da rede de cuidados à pessoa com deficiência.

Para a operacionalização dessa etapa, foi utilizada a ferramenta computacional denominada Poronto, que permite a extração de termos simples e termos compostos a partir de textos livres, e sua organização em uma lista por ordem de ocorrência ${ }^{(10)}$. Foi gerada uma relação de 2.778 termos com suas respectivas frequências de aparição, que foi exportada para uma planilha eletrônica. Os termos foram manualmente normalizados e uniformizados por gênero, número, sinonímia, tempo verbal, ortografia e exclusão de elementos de ligação e de termos relacionados a procedimentos e diagnósticos médicos, gerando uma lista de 446 termos.

$\mathrm{Na}$ segunda etapa, os termos identificados foram submetidos ao processo de mapeamento, por meio do qual foram comparados com os termos contidos na $\mathrm{CIPE}^{\oplus}$ versão $2015^{(11)}$, resultando em 333 termos constantes e 113 não constantes nessa terminologia, que constituíram o banco de termos do estudo.

$\mathrm{Na}$ terceira etapa, foram construídos os enunciados de diagnósticos de enfermagem, com base no banco de termos elaborado na etapa anterior e nas diretrizes do Conselho Internacional de Enfermeiros (CIE), consubstanciadas na norma ISO 18.104:2014 ${ }^{(12)}$. Além dessas diretrizes, foram considerados o julgamento clínico e a experiência 
dos autores na área de reabilitação em LM, os quais possibilitaram a análise da hipótese diagnóstica quanto à sua pertinência para a prática clínica.

$\mathrm{Na}$ quarta etapa, os enunciados de diagnósticos de enfermagem construídos foram mapeados aos conceitos pré-coordenados da CIPE ${ }^{\varpi}$ versão 2015, resultando em uma lista com diagnósticos de enfermagem constantes e não constantes nessa terminologia. Para os enunciados não constantes, foi realizado um processo de análise de equivalência com os conceitos da $\mathrm{CIPE}^{\circledast}$, utilizando os seguintes critérios: se o enunciado identificado é similar ao da CIPE; ${ }^{\circledR}$ se é mais abrangente; se é mais restrito; e se não existe concordância, ou seja, é um enunciado novo ${ }^{(13)}$.

$\mathrm{Na}$ quinta etapa, os enunciados de diagnósticos de enfermagem foram classificados de acordo com as subcategorias dos modos adaptativos, segundo o referencial teórico de Roy.

\section{Aspectos ÉtICOS}

Não houve necessidade de aprovação do estudo por Comitê de Ética em Pesquisa, visto que foi utilizada somente a literatura como fonte de dados.

\section{RESULTADOS}

Foram identificados 446 termos considerados relevantes para a prática clínica de enfermagem com a prioridade eleita, sendo $333(74,7 \%)$ termos constantes e $113(25,3 \%)$ não constantes na CIPE $^{\oplus}$ versão 2015. Esses termos foram distribuídos de acordo com o Modelo de Sete Eixos dessa classificação, resultando em: $82(18,4 \%)$ termos no eixo Ação; 12 (2,7\%) no eixo Cliente; $171(38,3 \%)$ no eixo Foco; 29 (6,5\%) no eixo Julgamento; $61(13,7 \%)$ no eixo Localização; 69 (15,5\%) no eixo Meios; e $22(4,9 \%)$ no Tempo.

Com base nesses termos e nas diretrizes do CIE, foram construídos 92 enunciados de diagnósticos de enfermagem, que foram mapeados com os conceitos pré-coordenados da CIPE $^{\circledast}$ versão 2015, resultando em $42(45,7 \%)$ enunciados iguais, $15(16,3 \%)$ similares, três $(3,3 \%)$ mais abrangentes, $20(21,7 \%)$ mais restritos, e $12(13,0 \%)$ sem concordância com os conceitos da classificação. Os enunciados classificados como iguais e similares foram considerados constantes. Assim, foram verificados $57(62,0 \%)$ diagnósticos de enfermagem constantes e $35(38,0 \%)$ não constantes na $\mathrm{CIPE}^{\circledR}$ versão 2015.

Os enunciados de diagnósticos de enfermagem foram categorizados de acordo com os modos adaptativos, segundo referencial teórico de Roy, e distribuídos da seguinte maneira: Modo Fisiológico: 66 (71,7\%) enunciados, Modo Papel Funcional: 12 (13,0\%) enunciados, Modo Autoconceito: 10 (10,9\%) enunciados e Modo Interdependência: quatro $(4,4 \%)$ enunciados - Quadros 1 e 2.

Quadro 1 - Distribuição dos enunciados de diagnósticos de enfermagem para pessoas com lesão medular em reabilitação, segundo o Modo Fisiológico - São Luís, MA, Brasil, 2017.

\begin{tabular}{|c|c|}
\hline \multicolumn{2}{|r|}{ MODO FISIOLÓGICO } \\
\hline Oxigenação & Respiração eficaz; Respiração prejudicada; Risco de função do sistema respiratório prejudicada. \\
\hline Nutrição & Ingestão de líquidos aumentada; Ingestão de líquidos diminuída; Peso corporal aumentado; Peso corporal diminuído. \\
\hline Eliminação & $\begin{array}{l}\text { Constipação, Incontinência intestinal, Incontinência urinária, Incontinência urinária de esforço, Incontinência urinária de } \\
\text { urgência, Micção prejudicada, Retenção urinária, Risco de constipação; Risco de retenção urinária. }\end{array}$ \\
\hline Atividade e repouso & $\begin{array}{c}\text { Amplitude de movimento prejudicada; Deformidade musculoesquelética presente; Capacidade para alimentar-se } \\
\text { prejudicada; Capacidade para aprontar-se prejudicada; Capacidade para realizar a higiene bucal prejudicada; Capacidade } \\
\text { para realizar a higiene corporal prejudicada; Capacidade para realizar o autocuidado prejudicada; Capacidade para se } \\
\text { transferir para a cadeira de rodas prejudicada; Capacidade para se transferir para a cama prejudicada; Capacidade para se } \\
\text { transferir para o carro prejudicada; Capacidade prejudicada para transpor barreiras ambientais; Capacidade prejudicada } \\
\text { para vestir e despir parte inferior do corpo; Capacidade prejudicada para vestir e despir parte superior do corpo; Marcha } \\
\text { prejudicada; Mobilidade na cadeira de rodas prejudicada; Mobilidade na cama prejudicada; Risco de deformidade } \\
\text { musculoesquelética. }\end{array}$ \\
\hline Proteção & $\begin{array}{c}\text { Febre; Higiene corporal prejudicada; Hipotensão postural; Infecção urinária; Lesão por pressão (especificar estágio e } \\
\text { localização); Lesão por queda (especificar localização); Lesão por transferência (especificar localização); Lesão térmica } \\
\text { (especificar localização); Pressão sanguínea alterada; Queda; Risco de complicação relacionada ao uso de órteses; Risco } \\
\text { de fratura; Risco de infecção (especificar); Risco de infecção urinária; Risco de lesão pelo uso de fralda; Risco de lesão por } \\
\text { pressão; Risco de lesão por queda; Risco de lesão por transferência; Risco de queda; Risco de trauma; Risco de trombose } \\
\text { venosa profunda. }\end{array}$ \\
\hline Sentidos & $\begin{array}{l}\text { Dor aguda (especificar intensidade e localização); Dor crônica (especificar intensidade e localização); Dor } \\
\text { musculoesquelética (especificar intensidade e localização); Dor neuropática (especificar intensidade e localização); } \\
\text { Sensibilidade ausente (especificar localização); Sensibilidade diminuída (especificar tipo e localização); Visão prejudicada. }\end{array}$ \\
\hline Fluidos e eletrólitos & Edema periférico (especificar grau e localização). \\
\hline Função neurológica & $\begin{array}{c}\text { Comunicação prejudicada, Disreflexia autonômica, Espasticidade (especificar intensidade e localização); Risco de } \\
\text { disreflexia autonômica. }\end{array}$ \\
\hline
\end{tabular}


Quadro 2 - Distribuição dos enunciados de diagnósticos de enfermagem para pessoas com lesão medular em reabilitação, segundo o Modos Papel Funcional, Autoconceito e Interdependência - São Luís, MA, Brasil, 2017.

\begin{tabular}{|c|c|c|c|c|}
\hline MODO PAPEL FUNCIONAL \\
\hline $\begin{array}{c}\text { Capacidade do cuidador para executar o cuidado prejudicada; Falta de conhecimento (especificar) sobre a lesão medula; Falta de conhecimento } \\
\text { sobre a medicação; Falta de conhecimento sobre as complicações da lesão medular; Falta de conhecimento sobre o manejo da bexiga } \\
\text { neurogênica; Falta de conhecimento sobre o manejo da dor neuropática; Falta de conhecimento sobre o manejo do intestino neurogênico; Falta de } \\
\text { conhecimento sobre o processo de reabilitação; Falta de conhecimento sobre política de saúde para pessoas com deficiência; Falta de capacidade } \\
\text { para manter a saúde; Falta de segurança no domicílio; Processo familiar prejudicado. }\end{array}$ \\
MODO AUTOCONCEITO \\
\hline $\begin{array}{c}\text { Ansiedade (especificar intensidade); Ansiedade relacionada à morte; Autoimagem negativa; Baixa autoestima; Disfunção sexual; Humor deprimido; } \\
\text { Padrão de sexualidade prejudicado; Risco de agressão física dirigida a terceiros; Risco de agressão verbal dirigida a terceiros; Risco de depressão. }\end{array}$ \\
\hline MODO INTERDEPENDÊNCIA \\
\hline Acesso ao serviço de saúde prejudicado; Isolamento social; Participação social prejudicada; Risco de isolamento social.
\end{tabular}

\section{DISCUSSÃO}

Os enunciados de diagnósticos de enfermagem elaborados foram mapeados de acordo com os modos adaptativos segundo Modelo de Adaptação de Roy. A maioria relacionou-se ao Modo Fisiológico, nas necessidades básicas associadas à oxigenação, nutrição, eliminação, atividade e repouso, e proteção, e nos processos complexos associados com os sentidos, fluidos e eletrólitos, e função neurológica.

A necessidade básica Oxigenação pode se encontrar afetada na pessoa com LM, principalmente acima do nível de T6, devido às complicações do sistema respiratório, como o comprometimento dos músculos expiratórios, reduzindo a efetividade da tosse para higiene brônquica. Esses pacientes apresentam um risco aumentado para atelectasias, infecções respiratórias e insuficiência respiratória, aumentando a suscetibilidade a hospitalizações e morte ${ }^{(14)}$. No contexto da reabilitação, devem ser desenvolvidas intervenções terapêuticas que auxiliem a mecânica muscular respiratória e o restabelecimento da eficácia da tosse, prevenindo complicações respiratórias ${ }^{(15)}$.

A ingestão adequada de nutrientes é de suma importância para a manutenção da saúde e melhor qualidade de vida, compreendida na necessidade básica Nutrição ${ }^{(4)}$. A pessoa com LM deve priorizar a ingestão de fibras e outros alimentos laxativos e realizar o controle da ingestão hídrica, a fim de favorecer o funcionamento intestinal e vesical, geralmente comprometidos pela lesão neurológica, e o processo de cicatrização de lesões ${ }^{(16)}$. Ressalta-se, também, que a perda progressiva ou o excesso de peso representam aspectos limitantes no processo de reabilitação desses indivíduos ${ }^{(17)}$.

Durante o processo de reabilitação, a abordagem nutricional é apoiada pelo profissional nutricionista, e o enfermeiro também exerce papel importante no monitoramento da adesão às orientações durante o período de internação hospitalar e nas consultas de acompanhamento ambulatorial, provendo respostas adaptativas para mudanças de hábitos alimentares no controle do peso, da glicemia, do colesterol e da pressão arterial, na prevenção de constipação intestinal e na cicatrização de feridas.

Como consequência da interrupção dos nervos da medula espinal, as mensagens provenientes da bexiga e/ou da porção retal para o cérebro não conseguem passar pelo bloqueio na altura da lesão, o que pode prejudicar a necessidade básica Eliminação. As alterações no padrão de funcionamento vesical podem levar ao quadro denominado bexiga neurogênica, caracterizado por arreflexia, hipocontratilidade ou hiperatividade detrusora, com perda ou redução da sensibilidade de repleção vesical e esvaziamento vesical incompleto, predispondo o paciente ao risco aumentado para infecção do trato urinário, litíase vesical, refluxo vesicoureteral e complicações renais $^{(18)}$. As alterações na motilidade intestinal dependem do nível e da extensão da lesão, e resultam em incontinência fecal e/ou constipação intestinal, podendo evoluir para complicações caso não sejam tratadas adequadamente ${ }^{(19)}$.

Compreendendo que as alterações vesicointestinais acarretam prejuízos de ordem física, social e emocional na vida da pessoa com LM, torna-se mandatório o início precoce do programa de reabilitação para esses sujeitos, com enfoque na adaptação à nova condição, ensino de técnicas para promover o esvaziamento vesical e intestinal adequado, reeducação alimentar e prevenção de complicações, a fim de minimizar os impactos na qualidade de vida e promover a reintegração social.

A necessidade básica Atividade e Repouso é responsável pelo equilíbrio dos processos básicos de mobilidade e de sono, essenciais para a função fisiológica ótima de todos os componentes do corpo e períodos de restauração e restabelecimento ${ }^{(4)}$. O comprometimento da mobilidade física na pessoa com LM é decorrente, sobretudo, da lesão neurológica que leva a alterações na função musculoesquelética em extensão variável, de acordo com nível e tipo de lesão, e pode acarretar múltiplas limitações funcionais, gerando diferentes graus de dependência para a realização das atividades da vida diária e o próprio autocuidado. Além disso, as sequelas da imobilidade determinam problemas sociais, econômicos e emocionais que precisam ser considerados durante o processo de reabilitação $0^{(1)}$.

A necessidade básica Proteção estabelece padrões relacionados com a integridade da pele e as defesas do organismo contra agentes ambientais ${ }^{(4)}$. Sabe-se que a pessoa com LM está vulnerável a lesões de pele desde a fase aguda da lesão até a reinserção comunitária, devido às alterações decorrentes da redução da mobilidade e sensibilidade abaixo do nível da lesão ${ }^{(19)}$. Nesses indivíduos, as lesões por pressão são a causa mais comum de complicação, cuja incidência varia entre 
$7,5 \%$ e $31,7 \%$ em unidades de reabilitação ${ }^{(20-21)}$, acarretando impacto negativo na qualidade de vida destes pacientes. As quedas também são um problema comum nessa população, ocorrem principalmente no momento das transferências, e correspondem a 90,0\% das complicações não infecciosas em pacientes com LM hospitalizados ${ }^{(22)}$. Com base nesses aspectos, cabe ao enfermeiro reabilitador desenvolver um plano de cuidados que vise a prevenção e o tratamento de complicações que possam resultar em atraso ou interrupção do processo de reabilitação desses pacientes.

O processo complexo Sentidos habilita as pessoas para interação com o meio ${ }^{(4)}$. Na pessoa com LM, as necessidades de percepção tátil, dolorosa, vibratória, de pressão e proprioceptiva podem ser experienciadas com alterações, devido às sequelas neurológicas ${ }^{(9)}$.

A dor após a LM é uma condição clínica de alta prevalência e difícil manejo, sendo considerada uma das complicações mais incapacitantes vivenciadas pelas pessoas no processo de reabilitação ${ }^{(23)}$. A dor pode ser classificada em nociceptiva ou neuropática. A dor nociceptiva geralmente está localizada no mesmo nível ou acima do nível neurológico da LM, sendo originada dos nociceptores somáticos ou viscerais. A dor neuropática é causada por uma lesão primária ou disfunção no sistema nervoso central, sendo geralmente caracterizada por alodínia, hiperestesias, hiperalgia, hiperpatia, parestesias e disestesias ${ }^{(24)}$. A dor afeta a capacidade de realizar as atividades cognitivas, sociais, recreativas e laborativas, além de interferir na realização das atividades de vida diária, acarretando impacto negativo sobre a qualidade de vida desses sujeitos. Nesse contexto, o programa de reabilitação do paciente com LM deve ser rigorosamente planejado por uma equipe interdisciplinar, visando o conhecimento e o controle da dor ${ }^{(25)}$.

O processo complexo Fluidos e Eletrólitos envolve a manutenção do equilíbrio entre os fluidos e os eletrólitos do organismo ${ }^{(4)}$. Devido à imobilidade e/ou à permanência prolongada na posição sentada, é comum a ocorrência de edema periférico em pessoas com LM, caracterizado pelo acúmulo de líquido no espaço intersticial, acometendo, principalmente, os membros inferiores ${ }^{(26)}$. Diante do exposto, é necessário prevenir e controlar o edema periférico nesses pacientes.

O processo complexo Função Neurológica pode se encontrar afetado em decorrência das alterações motoras, sensitivas e/ou autonômicas secundárias à LM, sendo necessário introduzir um programa de reabilitação complexo e individualizado com vistas à prevenção e tratamento precoce de complicações e obtenção de ganhos funcionais, de acordo com o nível de lesão e os potenciais do paciente ${ }^{(9)}$.

A espasticidade é um dos distúrbios mais frequentes e incapacitantes observado nas pessoas com LM. Caracteriza-se pelo aumento do tônus muscular (hipertonia), dos reflexos somáticos (hiperreflexia), clonus e espasmos dolorosos em resposta à hiperexcitabilidade do reflexo de estiramento. Este fator traz sérias consequências ao paciente, pode gerar incapacidade, impedir ou dificultar a realização das atividades de vida diária, além de causar contraturas e deformidades, e aumentar o risco para ocorrência de lesão por pressão e quedas ${ }^{(27)}$. Portanto, é mandatório que o enfermeiro, em conjunto com outros profissionais da equipe de reabilitação, avalie se a espasticidade é um fator limitante desse processo, visando seu controle e a prevenção de complicações.

A disreflexia autonômica é outra complicação clínica secundária à LM, caracterizada como uma crise hipertensiva, geralmente acompanhada por bradicardia, cefaleia intensa, piloereção, sudorese profusa, dilatação das pupilas e rubor cutâneo, desencadeada por estímulos nociceptivos ou viscerais abaixo do nível da lesão. Ocorre comumente em pacientes com LM acima do nível T6. Inúmeros estímulos podem desencadear este reflexo, como a distensão de órgãos viscerais (bexiga e intestino), lesões de pele, pressão nos segmentos corporais sem sensibilidade protetora, paroníquia, dentre outros. $\mathrm{O}$ tratamento consiste na identificação do estímulo nocivo e sua eliminação, e é responsabilidade do enfermeiro reabilitador ensinar e educar o paciente/família/cuidador para o reconhecimento, prevenção e controle dessa complicação ${ }^{(27)}$.

Ressalta-se, no presente estudo, que os aspectos psicossociais também foram contemplados com enunciados de diagnósticos de enfermagem, o que configura a possibilidade do atendimento da pessoa com LM em todos os fatores que influenciam sua adaptação durante o processo de reabilitação.

O Modo Papel Funcional incide sobre os aspectos sociais relacionados aos papéis que a pessoa ocupa na sociedade ${ }^{(4)}$. Durante o programa de reabilitação, os profissionais da equipe interdisciplinar de saúde, dentre eles o enfermeiro, executam ações de educação, prevenção e promoção da saúde a pacientes, cuidadores e familiares, criando condições favoráveis à manutenção da funcionalidade, objetivando a reinserção social da pessoa com LM. Para isso, o indivíduo precisa atribuir novos significados à sua existência a fim de adaptar-se à sua condição atual ${ }^{(27)}$. Entretanto, alguns comportamentos, como não aceitação da nova condição, falta de conhecimento do prognóstico e do tratamento ou dificuldade de aderir às orientações, podem ser considerados limitantes desse processo.

O Modo Autoconceito engloba aspectos psicológicos e espirituais que a pessoa mantém sobre ela mesma, e divide-se em dois componentes: eu físico (abrange a sensação e a autoimagem corporal) e o eu pessoal (engloba o eu consistência, o eu ideal e o eu ético-moral-espiritual) $)^{(4)}$. As inúmeras sequelas de uma LM impactam na autoimagem, no reconhecimento da nova condição física e nas relações interpessoais e com ambiente em seu entorno. Isso pode gerar raiva, insegurança, medo, sentimento de inferioridade, desesperança, baixa autoestima, dentre outros. $\mathrm{O}$ enfermeiro e demais profissionais da equipe multidisciplinar devem estar preparados para proporcionar apoio emocional aos pacientes e auxiliá-los no enfrentamento de sua nova condição em todo o processo de reabilitação ${ }^{(28)}$.

O Modo Interdependência enfoca interações relacionadas a dar e receber afeto, respeito e valor, e abrange as relações interativas mantidas entre a pessoa e os outros, na tentativa de satisfazer as suas necessidades ${ }^{(4)}$. $\mathrm{O}$ advento da LM transforma significativamente a vida dos indivíduos 
nos mais variados aspectos, o que pode levar ao isolamento social pela dificuldade de expressar emoções e enfrentar a nova condição. Durante o processo de reabilitação em LM, buscam-se estratégias que auxiliem na adaptação do individuo à situação, estimulando sua participação social, o envolvimento da família e a ampliação das redes de apoio. Isso contribui de maneira significativa para reintroduzir a pessoa com LM na vida familiar e social( ${ }^{(29)}$.

O presente estudo apresenta limitações, como a ausência de indicadores para a construção de diagnósticos de enfermagem relacionados ao sono (compreendido na necessidade básica Atividade e Repouso) e ao processo complexo Função Endócrina. Esse fato precisa ser corrigido, pois esses componentes são fundamentais para produzir uma função fisiológica ótima de todo organismo e, portanto, devem ser considerados no planejamento dos cuidados pelo enfermeiro reabilitador. Destaca-se também que o banco de termos e os diagnósticos de enfermagem construídos não foram validados por especialistas, suscitando a importância de estudos adicionais com o objetivo de promover a validação de conteúdo e verificação de sua aplicabilidade na prática clínica.

Contudo, o produto desta investigação poderá nortear o raciocínio crítico e julgamento clínico do enfermeiro no planejamento das intervenções de enfermagem, mediante uso de uma linguagem uniformizada, contribuindo para a efetiva aplicação do processo de enfermagem na reabilitação de pessoas com LM em todas as suas etapas. Desse modo, sugere-se, no seguimento da pesquisa, a construção de enunciados de intervenções de enfermagem para estruturar um subconjunto terminológico da CIPE ${ }^{\circledast}$, visando operacionalizar a sistematização da assistência de enfermagem no contexto da reabilitação, viabilizar a documentação das práticas de cuidado, e facilitar a comunicação entre os enfermeiros, levando a benefícios importantes para a profissão.

\section{CONCLUSÃO}

Os enunciados de diagnósticos de enfermagem elaborados retratam focos de atenção para o cuidado de enfermagem a pessoas com LM em reabilitação, a partir de dados evidenciados em documentação especializada, com base em referencial teórico de enfermagem e em linguagem padronizada, contribuindo para direcionar as ações da assistência de enfermagem de forma sistematizada, individualizada e resolutiva. Outros diagnósticos podem ser incluídos nessa nomenclatura, a partir das demandas de necessidades individuais de cada pessoa.

\section{RESUMO}

Objetivo: Construir enunciados de diagnósticos de enfermagem da Classificação Internacional para a Prática de Enfermagem (CIPE ${ }^{\circledast}$ para a especialidade de reabilitação em lesão medular, com base no Modelo de Adaptação de Callista Roy. Método: Pesquisa descritiva desenvolvida em etapas sequenciadas de identificação e mapeamento cruzado de termos relevantes para o cuidado com a prioridade de saúde, construção e mapeamento cruzado de enunciados de diagnósticos de enfermagem, com base na $\mathrm{CIPE}^{\circledast}$, e categorização dos diagnósticos segundo os modos adaptativos. Resultados: Foram elaborados 92 enunciados de diagnósticos de enfermagem, dos quais 66 foram classificados no Modo Fisiológico, 12 no Modo Papel Funcional, 10 no Modo Autoconceito e quatro no Modo Interdependência. Conclusão: Os diagnósticos de enfermagem elaborados retratam focos de atenção para o cuidado de enfermagem a pessoas com lesão medular em reabilitação, contribuindo para o direcionamento das ações da assistência de enfermagem de forma sistematizada, individualizada e resolutiva.

\section{DESCRITORES}

Enfermagem; Diagnóstico de Enfermagem; Terminologia; Classificação; Reabilitação; Traumatismos da Medula Espinal.

\section{RESUMEN}

Objetivo: Construir enunciados de diagnóstico de enfermería de la Clasificación Internacional para la Práctica de Enfermería $\left(\mathrm{CIPE}^{\circledast}\right)$ para la especialidad de rehabilitación de la médula espinal, basados en el Modelo de Adaptación de Callista Roy. Método: Estudio descriptivo desarrollado en pasos secuenciales de identificación y mapeo cruzado de términos relevantes para el cuidado con la prioridad de salud, construcción y mapeo cruzado de enunciados de diagnóstico de enfermería con base en la CIPE ${ }^{\oplus}$ y la categorización de diagnósticos según modos adaptativos. Resultados: Se desarrollaron 92 enunciados de diagnósticos de enfermería, de los cuales 66 se clasificaron en Modo Fisiológico, 12 en Modo Rol Funcional, diez en Modo Autoconcepto y cuatro en Modo Interdependencia. Conclusión: Los diagnósticos de enfermería desarrollados retratan focos de atención para el cuidado de enfermería a personas con lesión medular en rehabilitación, contribuyendo a la dirección de las acciones de cuidados de enfermería de manera sistemática, individualizada y resolutiva.

\section{DESCRIPTORES}

Enfermería; Diagnóstico de Enfermería; Terminología; Clasificación; Rehabilitación; Traumatismos de la Médula Espinal.

\section{REFERÊNCIAS}

1. Prudente CO, Ribeiro MF, Porto CC. [Quality of life of family caregivers of adults with spinal cord injury: a systematic review]. Cienc Saúde Coletiva. 2017;22(1):123-34. Portuguese. https://doi.org/10.1590/1413-81232017221.08072015

2. Clares JW, Fernandes BK, Guedes MV, Freitas MC. Specialized nursing terminology for the care of people with spinal cord injury. Rev Esc Enferm USP. 2019;53:e03445. https://doi.org/10.1590/s1980-220x2018014203445

3. Carvalho CM, Cubas MR, Nóbrega MM. Brazilian method for the development terminological subsets of ICNPR: limits and potentialities. Rev Bras Enferm. 2017;70(2):430-5. https://doi.org/10.1590/0034-7167-2016-0308

4. Roy C, Andrews HA. The Roy adaptation model. Lisboa: Instituto Piaget; 2001.

5. Medeiros LP, Souza MB, Sena JF, Melo MD, Costa JW, Costa IK. [Roy Adaptation Model: integrative review of studies conducted in the light of the theory]. Rev Rene. 2015;16(1):132-40. Portuguese. https://doi.org/10.15253/2175-6783.2015000100017 
6. Andrade LT, Araújo EG, Andrade KR, Soares DM, Chianca TC. [Role of nursing in physical rehabilitation]. Rev Bras Enferm. 2010;63(6):1056-60. Portuguese. https://doi.org/10.1590/S0034-71672010000600029

7. Coenen A, Kim TY. Development of terminology subsets using ICNP. Int J Med Inform. 2010 Jul;79(7):530-8. https://doi.org/10.1016/j. ijmedinf.2010.03.005

8. International Council of Nurses. Guidelines for ICNP® catalogue development [Internet]. Geneve: International Council of Nurses; 2008 [cited 2018 Apr 20]. Available from: http://www.icn.ch/images/stories/documents/programs/icnp/icnp_catalogue_development.pdf

9. Brasil. Ministério da Saúde. Secretaria de Atenção à Saúde. Departamento de Ações Programáticas Estratégicas. Diretrizes de atenção à pessoa com lesão medular [Internet]. Brasília, DF: Ministério da Saúde; 2015 [cited 2020 Dec 11]. Availabe from: http://bvsms.saude.gov. br/bvs/publicacoes/diretrizes_atencao_pessoa_lesao_medular_2ed.pdf

10. Zahra FM, Carvalho DR, Malucelli A. [Poronto: tool for semi-automatic ontology construction in portuguese]. J Health Inform [Internet]. 2013 [cited 2016 Oct 16];5(2):52-9. Available from: http://www.jhi-sbis.saude.ws/ojs-jhi/index.php/jhi-sbis/article/view/232/167

11. Garcia TR. Classificação internacional para prática da enfermagem - CIPE®: aplicação à realidade brasileira. Porto Alegre: Artmed; 2015.

12. Marin HF, Peres HH, Dal Sasso GT. Categorical structure analysis of ISO 18104 standard in nursing documentation. Acta Paul Enferm. 2013;26(3):299-306. https://doi.org/10.1590/S0103-21002013000300016

13. Leal MT. CIPE® e a visibilidade da enfermagem; mitos e realidades. Loures: Lusociência; 2006.

14. Berlowitz DJ, Wadsworth B, Ross J. Respiratory problems and management in people with spinal cord injury. Breathe (Sheff). 2016;12(4):328-40. https://doi.org/10.1183/20734735.012616

15. Macedo FS, Paz CC, Rocha AF, Miosso CJ, Carvalho HB, Mateus SR. New perspectives for chest physical therapy in spinal cord injury: a systematic review. Acta Paul Enferm. 2017;30(5):554-64. https://doi.org/10.1590/1982-0194201700077

16. Khalil RE, Gorgey AS, Janisko M, Dolbow DR, Moore JR, Gater DR. The role of nutrition in health status after spinal cord injury [Internet]. Aging Dis. 2013 [cited 2017 Jan 17];4(1):14-22. Available from: https://www.ncbi.nlm.nih.gov/pmc/articles/PMC3570137/ pdf/ad-4-1-14.pdf

17. Abreu T, Friedman R, Fayh AP. [Pathophysiology and nutritional status assessment of individuals with physical disabilities]. Rev HCPA [Internet]. 2011 [cited 2020 Jan 12];31(3):345-52. Available from: http://seer.ufrgs.br/index.php/hcpa/article/view/17907/13952

18. Marialva C, Bettencourt M, Vale P, Bastos J, Carvalho M, Faria F, et al. Efficacy of botulinum toxin type A in the treatment of neurogenic lower urinary tract disfunction due to spinal cord injury. Acta Urol Port [Internet]. 2015 [cited 2020 Jun 26];32:113-7. Available from: https://www.scipedia.com/public/Marialva_et_al_2015a

19. Campoy LT, Rabeh SA, Castro FF, Nogueira PC, Terçariol CA. Bowel rehabilitation of individuals with spinal cord injury: video production Rev Bras Enferm. 2018;71(5):2376-82. https://doi.org/10.1590/0034-7167-2017-0283

20. Dejong G, Hsieh CH, Brown P, Smout RJ, Horn SD, Ballard P, et al. Factors associated with pressure ulcer risk in spinal cord injury rehabilitation. Am J Phys Med Rehabil. 2014;93(11):971-86. https://doi.org/10.1097/PHM.0000000000000117

21. New PW, Rawicki HB, Bailey MJ. Nontraumatic spinal cord injury rehabilitation: pressure ulcer patterns, prediction, and impact. Arch Phys Med Rehabil. 2004;85(1):87-93. https://doi.org/10.1016/S0003-9993(03)00378-2

22. Brotherton SS, Krause JS, Nietert PJ. Falls in individuals with incomplete spinal cord injury. Spinal Cord. 2007;45(1):37-40. https://doi.org/10.1038/sj.sc.3101909

23. Loh E, Guy SD, Mehta S, Moulin DE, Bryce TN, Middleton JW, et al. The CanPain SCl clinical practice guidelines for rehabilitation management of neuropathic pain after spinal cord: introduction, methodology and recommendation overview. Spinal Cord. 2016;54(S1 Suppl 1):S1-6. https://doi.org/10.1038/sc.2016.88

24. Laluce TO, Dalul CM, Martins MR, Ribeiro RC, Almeida FC, Cesarino CB. Coping strategies in patients with neuropathic pain. BrJP. 2019;2(3):260-6. https://doi.org/10.5935/2595-0118.20190046

25. Rodrigues AV, Vidal WA, Lemes JA, Gôngora CS, Neves TC, Santos SM, et al. Study on the characteristics of pain in patients with spinal cord injury. Acta Fisiatr. 2012;19(3):171-7. https://doi.org/10.5935/0104-7795.20120027

26. Guzelkucuk U, Duman I, Yılmaz B, Tan AK. Reversible post-pregabalin peripheral edema in a spinal cord injury patient. Spinal Cord. 2012;50(6):472-3. https://doi.org/10.1038/sc.2011.79

27. Rabchevsky AG, Patel SP, Duale H, Lyttle TS, O'Dell CR, Kitzman PH. Gabapentin for spasticity and autonomic dysreflexia after severe spinal cord injury. Spinal Cord. 2011;49(1):99-105. https://doi.org/10.1038/sc.2010.67

28. Amaral MT. A new sense for living: a comprehensive study about the adaptation process following spinal cord injuries. Rev Esc Enferm USP. 2009;43(3):573-80. https://doi.org/10.1590/S0080-62342009000300011

29. Vasco CC, Franco MH. [Paraplegic individuals and the meaning-making process of the spinal cord injury in their lives]. Psicol Cienc Prof. 2017;37(1):119-31. https://doi.org/10.1590/1982-3703000072016 